\title{
Linkage disequilibrium between the M470V variant and the IVS8 polyT alleles of the CFTR gene in CBAVD
}

Anne de Meeus, Caroline Guittard, Marie Desgeorges, Soukeyna Carles, Jacques Demaille, Mireille Claustres

\begin{abstract}
Congenital bilateral absence of the vas deferens (CBAVD) is a cause of male sterility mostly resulting from mutations in the cystic fibrosis transmembrane regulator (CFTR) gene. The most common defect is the $5 \mathrm{~T}$ variant at the branch/ acceptor site of intron 8 , which induces high levels of exon 9 skipping leading to non-functional protein. However, this 5T variant has incomplete penetrance and variable expressivity, suggesting that some other regulatory factors may modulate the splicing of exon 9. To identify such factors, we report here the genetic analysis of a polymorphic locus, M470V, located in exon 10 of the CFTR gene in 60 patients with CBAVD, compared to a normal control population. The statistical analysis showed strong linkage disequilibrium between the 5T allele and the $V$ allele of the M470V polymorphism in the CBAVD population, but not in the normal population. The $V$ allele in a gene carrying 5T could, however, contribute to lowering the level of normal transcripts, as already suggested by in vitro transcriptional studies. These genetic findings, together with previous studies, suggest involvement of the M470V variant in the modulation of the splicing of exon 9 of the CFTR gene. (F Med Genet 1998;35:594-596)
\end{abstract}

Keywords: M470V; CFTR gene; CBAVD

Cystic fibrosis (CF) is a severe autosomal recessive disease caused by mutations in the cystic fibrosis transmembrane regulator (CFTR) gene. ${ }^{1-3}$ The disease shows a highly variable clinical presentation. Genotype- exon 9 skipping compared to the $9 \mathrm{~T}$ and $7 \mathrm{~T}$ alleles, leading to non-functional protein. ${ }^{7}$ Thus, the $5 \mathrm{~T}$ allele is now considered as a mutation, even with partial penetrance and great variability in disease expression. The first molecular basis of partial penetrance of this splicing mutation has been published recently by Rave-Harel et $a l^{8}{ }^{8}$ who reported great variability in the efficiency of the splicing mechanism among distinct organs of one subject and between subjects as well. They established a correlation between the level of correctly spliced RNA transcribed from the gene carrying the $5 \mathrm{~T}$ allele and disease expression in both respiratory and epididymal epithelium. Furthermore, Teng et al reported that the proportion of transcripts lacking exon 9 was increased in vas deferens cells compared with nasal epithelial cells, independently of the intron $8 \mathrm{Tn}$ genotype. These results suggested that the level of normal transcripts depended on both intron $8 \mathrm{Tn}$ allele and tissue, in which different regulation levels were observed, thus indicating the involvement of trans acting factors. In other respects, the exon $10 \mathrm{M} 470 \mathrm{~V}$ locus is the most polymorphic two allele locus of the CFTR gene. In 1996, Cuppens et $a l^{10}$ reported an increased proportion of mRNA lacking exon 9 when transcribed from a CFTR gene carrying the $\mathrm{V}$ allele. They further showed by in vitro studies that the $\mathrm{V}$ allele yielded a lower functional CFTR protein rate, at both transcriptional and translational levels, independently of the intron $8 \mathrm{Tn}$ genotype. The aim of our study was to elucidate if the observed influence of the M470V locus on exon 9 skipping in vas deferens cells was restricted to the molecular level or if it could have any pathological consequences.

We analysed the intron $8 \mathrm{Tn}$ tract $^{5}$ and the M470V locus ${ }^{11}$ in 64 unrelated CBAVD patients comprising 24 carrying two mutations in the CFTR gene, 18 carrying one mutation and the $5 \mathrm{~T}$ allele, seven carrying one mutation, two carrying the $5 \mathrm{~T}$ allele, one homozygote for the $5 \mathrm{~T}$ allele, and 12 carrying no mutation. ${ }^{12} \mathrm{~A}$ total of 266 normal chromosomes from normal related subjects and parents of CF children were used as a control group. Statistical analysis was performed using GENEPOP (version 3.0) software. ${ }^{13}$

Haplotypes could be established for only 60 of the 64 patients (table 1), who were thus included in the statistical analysis. The 5T allele was found on 20 chromosomes in the non- $\Delta$ F508 group of chromosomes and was \footnotetext{
phenotype correlations have been established for some mutations, although substantial variability in disease expression can still be observed among patients carrying the same mutation. Almost all CF men are infertile as a result of congenital absence of the vas deferens (CBAVD). CBAVD can also be found in otherwise healthy men. The high frequency of CFTR mutations among men with CBAVD indicates that isolated CBAVD could be an incomplete form of $\mathrm{CF}^{4}$ The $5 \mathrm{~T}$ variant at the branch/acceptor site of intron 8 is the most common CFTR defect in CBAVD patients. ${ }^{56}$ This site has three alleles with nine, seven, and five thymidines (9T, 7T, 5T, respectively). The

$5 \mathrm{~T}$ allele was found to induce higher levels of

Received 4 July 1997 Revised version accepted for publication 15 December 1997

Biochimie Génétique, Montpellier, France

M Desgeorges

J Demaille

Correspondence to:

Dr Claustres.
} 
Table 1 Frequencies of the intron $8(T) n-M 470 V$ haplotypes in CBAVD and normal chromosomes

\begin{tabular}{|c|c|c|c|c|c|c|c|}
\hline & \multicolumn{7}{|c|}{ Haplotypes } \\
\hline & $9-M$ & $9-V$ & $7-M$ & $7-V$ & $5-M$ & $5-V$ & Total \\
\hline \multicolumn{8}{|l|}{ CBAVD chromosomes } \\
\hline$\Delta \mathrm{F} 508$ & 27 & 2 & 0 & 0 & 0 & 0 & 29 \\
\hline Non- $\Delta F 508$ & 10 & 3 & 27 & 31 & 4 & 16 & 91 \\
\hline Total & 37 & 5 & 27 & 31 & 4 & 16 & 120 \\
\hline Normal chromosomes & 16 & 7 & 85 & 147 & 7 & 4 & 266 \\
\hline
\end{tabular}

not found associated in cis with an identified mutation. The aim of the statistical analysis of the genotypic results was to detect if the genotypic distribution was significantly different between CBAVD and normal populations. Analysis of allele frequencies at both the Tn and M470V loci showed a non-identical allelic distribution in CBAVD and normal populations (Fisher's exact test, $\mathrm{p}<10^{-4}$ and $\mathrm{p}=0.004$ respectively). Since 29/120 (24\%) CBAVD chromosomes carried the $\Delta \mathrm{F} 508$ mutation, which is in complete linkage disequilibrium with $9 \mathrm{~T}$ and $M$ alleles, a subgroup of CBAVD chromosomes that did not carry the $\Delta \mathrm{F} 508$ mutation (CBAVD $\triangle F-$ ) was analysed in the same way. The distribution of Tn locus alleles remained significantly different from the normal group (Fisher's exact test, $\mathrm{p}<10^{-4}$ ). By contrast, frequencies of $M$ and $V$ alleles were not different from the control group (Fisher's exact test, $\mathrm{p}=0.46$ ), showing that the difference previously observed in the allelic distribution at the M470V locus was mostly the result of the high frequency of the $\Delta \mathrm{F} 508$ mutation in the CBAVD group. To analyse the linkage disequilibrium hypothesis against independence for both the Tn and M470V loci, we tested the three groups of chromosomes (CBAVD, CBAVD $\triangle \mathrm{F}$-, normal). We found linkage disequilibrium in the three populations (Fisher's exact test, CBAVD: $p<10^{-4}$, CBAVD $\triangle \mathrm{F}-$ : $p=0.00015$, normal: $p=0.0023$ ). Furthermore, we performed pairwise linkage disequilibrium analysis using the Linkdos program in GENEPOP ${ }^{14}$ in order to measure the effect for each allele (table 2). Positive linkage disequilibrium was detected between $9 \mathrm{~T}$ and $M$ alleles in the three populations and between $7 \mathrm{~T}$ and $\mathrm{V}$ alleles in the CBAVD and the control groups; it was not significant in the CBAVD $\triangle \mathrm{F}$ - group. The most striking result concerned the $5 \mathrm{~T}$ allele. In contrast with the normal population in which no significant association was found between $5 \mathrm{~T}$ and $\mathrm{M} 470 \mathrm{~V}$ alleles, the $5 \mathrm{~T}$ allele was found to be strongly associated with the $\mathrm{V}$ allele in both groups of CBAVD chromosomes.

Table 2 Analysis of linkage disequilibrium between Tn and M470 locus in the CBAVD, $C B A V D, \triangle F 508-$, and normal groups with Linkdos propram

\begin{tabular}{|c|c|c|c|c|c|c|}
\hline \multirow[b]{2}{*}{ Alleles } & \multicolumn{2}{|c|}{$C B A V D$} & \multicolumn{2}{|c|}{$C B A V D \triangle F 508-$} & \multicolumn{2}{|c|}{ Normal } \\
\hline & $L D$ & p value & $L D$ & p value & $L D$ & $p$ value \\
\hline 9T-M & $(+)$ & 0.0001 & $(+)$ & 0.0117 & $(+)$ & 0.0030 \\
\hline $9 \mathrm{~T}-\mathrm{V}$ & $(-)$ & 0.0001 & $(-)$ & 0.0117 & $(-)$ & 0.0030 \\
\hline 7T-M & $(-)$ & 0.0292 & (0) & 0.7005 & $(-)$ & 0.0006 \\
\hline $7 \mathrm{~T}-\mathrm{V}$ & $(+)$ & 0.0292 & (0) & 0.7005 & $(+)$ & 0.0006 \\
\hline 5T-M & $(-)$ & 0.0003 & $(-)$ & 0.0099 & (0) & 0.1107 \\
\hline $5 \mathrm{~T}-\mathrm{V}$ & $(+)$ & 0.0003 & $(+)$ & 0.0099 & (0) & 0.1107 \\
\hline
\end{tabular}

$\mathrm{LD}=$ means linkage disequilibrium. (+) Association and (-) lack of association between alleles, $(0)$ indicates absence of $\mathrm{LD}$.
This positive linkage disequilibrium between $5 \mathrm{~T}$ and $\mathrm{V}$ alleles in a CBAVD population has not been reported previously and suggests that the $M 470 \mathrm{~V}$ polymorphism could contribute to the variable expressivity of the $5 \mathrm{~T}$ allele.

The $\mathrm{V}$ allele cannot be considered as a disease predisposing factor by itself since its frequency is not increased in the CBAVD population. Observation of linkage disequilibrium between the $5 \mathrm{~T}$ and $\mathrm{V}$ alleles in the CBAVD population suggests additional effects of both alleles in the severity of the phenotypic expression. Such genetic results are in complete agreement with those obtained at the molecular level. Indeed, it has been shown that (1) the disease expression was correlated with the normal transcript level; CBAVD patients thus have low levels of correctly spliced RNA in the vas deferens, and (2) the rate of exon 9 skipping depended both on $\mathrm{Tn}$ tract $^{7}$ and M470V alleles ${ }^{10}$ and on tissue. ${ }^{89}$ The $\mathrm{V}$ allele in a $5 \mathrm{~T}$ carrying gene should thus aggravate the effect of the $5 \mathrm{~T}$ allele on the splicing mechanism defect and contribute to even lower levels of normal transcripts. In the study of Rave-Harel et al, ${ }^{8}$ however, the level of normal CFTR transcripts was no different between the $\mathrm{M}$ and $\mathrm{V}$ alleles in the respiratory epithelium, but no data concerning the epididymal epithelium were shown. As the studies of both Teng et $a l^{p}$ and Rave-Harel et al strongly suggest different regulation levels in both tissues, the observation of an equivalent rate of normal transcripts for the $\mathrm{M}$ and $\mathrm{V}$ alleles in respiratory tissue does not apply equally to epididymal tissue. Furthermore, the high level of mRNA lacking exon 9 in vas deferens cells reported by Teng et al ${ }^{9}$ suggested that those cells could be more sensitive to additional factors such as the $V$ allele that may contribute to the decreased levels of normal transcripts. It has been shown that the $\mathrm{V}$ allele increases the proportion of transcripts lacking exon $9^{10}$ and could thus be considered as a cis acting factor that contributes to decreasing the level of normal transcripts below the critical level which induces phenotypic expression.

Linkage disequilibrium alone is not a strong argument for a role of the $\mathrm{M} 470 \mathrm{~V}$ locus, since it could just indicate a third region close to this locus that might be implicated in exon 9 skipping regulation. However, no other polymorphism shows such linkage disequilibrium either in intron 8 or in exons 9 or 10. Thus, the M470V locus, so far considered an innocent polymorphism, should now be regarded as a possible modulating factor of CFTR expression.

We are indebted to Dr Michel Raymond for help in the statistical analysis using GENEPOP. We thank Dr Jamal Tazi for very helpful scientific discussion and Drs Patrice Bouvagnet and Isabelle Touitou for useful comments and suggestions.

1 Kerem E, Rommens J, Buchanan JA, et al. Identification of the cystic fibrosis gene: genetic analysis. Science 1989;245:1073-80.

2 Riordan JR, Rommens J, Kerem E, et al. Identification of the cystic fibrosis gene: cloning and characterization of complementary DNA. Science 1989;245:1066-73.

3 Rommens J, Iannuzzi MC, Kerem B, et al. Identification of the cystic fibrosis gene: chromosome walking and jumping. Science 1989;245:1059-65. 
4 Kerem B, Kerem E. The molecular basis for disease variability in cystic fibrosis. Eur f Hum Genet 1996;4:65-73. Chillon M, Casals T, Mercier B, et al. Mutations in the cystic fibrosis gene in patients with congenital absence of

6 Jarvi K, Zielenski J, Wilschanski M, et al. Cystic fibrosis transmembrane conductance regulator and obstructive azoospermia. Lancet 1995;345:1578.

7 Chu CS, Trapnell BC, Curristin S, Cutting GR, Crystal RG. Genetic basis of variable exon 9 skipping in cystic fibrosis transmembrane conductance regulator mRNA. Nat Genet 1993;3:151-6.

8 Rave-Harel N, Kerem E, Nissim-Rafinia $M$, et al. The molecular basis of partial penetrance of splicing mutations in cystic fibrosis. Am 7 Hum Genet 1997;60:87-94.

9 Teng $\mathrm{H}$, Jorissen $\mathrm{M}$, Van Poppel H, Legius E, Cassiman JJ, Cuppens H. Increased proportion of exon 9 alternatively spliced CFTR transcripts in vas deferens compared with nasal epithelial cells. Hum Mol Genet 1997;6:85-90.
10 Cuppens $H$, Teng $H$, Jaspers $M$, Willems $T$, Jorissen $M$, Cassiman JJ. Functional characterisation of the M470V ocus within the CFTR gene. Isr F Med Sci 1996;32(suppl):

11 Chehab FF, Johnson J, Louie E, Goossens M, Kawasaki E, Erlich H. A dimorphic 4-bp repeat in the cystic fibrosis gene is in absolute disequilibrium with the $\Delta \mathrm{F} 508$ mutation: implications for prenatal diagnosis and mutation origin. Am $\mathcal{F}$ Hum Genet 1991;48:223-6.

2 de Meeus A, Guittard C, Desgeorges M, Carles S, Demaille $J$, Claustres $M$. Genetic findings in congenital bilateral aplasia of vas deferens patients and identification of six novel mutations. Hum Mutat (in press)

13 Raymond M, Rousset F. GENEPOP (version 1.2): population genetics software for exact tests and ecumenicism. $\mathcal{F}$ Hered 1995;86:248-9.

14 Garnier-Gere P, Dillmann C. A computer program for testing pairwise linkage disequilibria in subdivided populations. F Hered 1992;83:248-9. $\frac{\mathfrak{c}}{\stackrel{2}{2}}$

ه

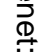

$\overrightarrow{\overrightarrow{\mathrm{S}}}$

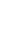

